\title{
A Study on Prototype Hybrid (LTS/HTS) Magnet for NMR Application
}

\author{
Sukjin Choi, Young Jin Hwang, and Tae Kuk Ko* \\ Yonsei University, Seoul 120-749, Korea
}

(Received 20 July 2011, Received in final form 30 August 2011, Accepted 2 September 2011)

\begin{abstract}
NMR over $1 \mathrm{GHz}(23.5 \mathrm{~T})$ level has difficulties in design and fabrication with only low temperature superconducting (LTS) wire because of its material characteristics such as the decay of critical current under the magnetic field. Because High temperature superconducting (HTS) tape has a good performance under the extremely high magnetic field, it has been developed for high-field magnet over $23.5 \mathrm{~T}$. In this paper, the LTS magnet was made for applying magnetic fields externally and the HTS coil was designed and fabricated. The electromagnetic field analysis has been done with respect to the structure and the operating current of the LTS and HTS coil. Considering to the field homogeneity and the center field, the design parameters which is suitable for the HTS coil were found. The HTS insert coil was impregnated with epoxy resin in order to prevent the movement of winding during energizing the magnet. The hybrid magnet (LTS/HTS) magnet was fabricated and tested based on the design parameters. The experimental result shows that the LTS background magnet and the HTS insert coil can be operated stable beyond $220 \mathrm{~A}$ and $210 \mathrm{~A}$. The final value $4.32 \mathrm{~T}$ at the center was acquired.
\end{abstract}

Keywords : homogeneity, HTS insert coil, LTS background magnet

\section{Introduction}

Nuclear magnetic resonance (NMR) is a property that magnetic nuclei have in a magnetic field and applied electromagnetic pulse or pulses, which cause the nuclei to absorb energy from the electromagnetic pulse and radiate this energy back out. Many scientific techniques exploit NMR phenomena to study molecular physics, crystals and non-crystalline materials through NMR spectroscopy.

NMR over $1 \mathrm{GHz}(23.5 \mathrm{~T})$ level, for research on the structure of polymer proteins, which is indispensable for advance in biotechnology has difficulties in design and fabrication with only low temperature superconductor (LTS) due to their material limitations. Therefore, the high temperature superconducting (HTS) coil technology, which has a good performance under the extremely high magnetic field, is necessarily required for the development of ultra high-field magnet over $23.5 \mathrm{~T}$ [1-3].

In this paper, the LTS background magnet for applying magnetic fields externally was made and the HTS coil was designed and fabricated. Considering the field homogeneity and the center field, the specification for the HTS

*Corresponding author: Tel: +82-2-2123-2772

Fax:+82-2-393-2834, e-mail: tkko@yonsei.ac.kr coil was derived. The HTS coil was fabricated using the designed specifications. The HTS coil was impregnated with an epoxy resin during the winding process (wet winding method). The stability of HTS is much higher than that of LTS, thus HTS magnets are hardly quenched. In the case that quench would occur in an HTS magnet, however, it seems to be hard to protect the HTS from overheating because it has very low quench propagation velocity along the wire and thus an HTS magnet is likely to burn out. So, the HTS magnet is operated at lower current than the critical current for the stable operating condition [3-5].

In this paper, we want to know whether or not the fabricated HTS insert coil can be operated stable in background magnetic fields. And the design margin of HTS magnet is evaluated considering this result. The numerical and experimental procedures are described and both results are discussed.

\section{Design and Fabrication}

\subsection{LTS background magnet}

The LTS background magnet for applying magnetic fields externally was designed and fabricated. The structure of a superconducting magnet is basically decided con- 
Table 1. Specification of LTS wire for LTS background magnet.

\begin{tabular}{ccc}
\hline \hline Specification & Value & Unit \\
\hline Manufacturer & Oxford Instrument & \\
Material & NbTi enamel/braided conductor & \\
\# of Filaments & 54 & \\
Ic @6 T & $>800$ & $\mathrm{~A}$ \\
Bare Wire Diameter & 1.10 & $\mathrm{~mm}$ \\
Inner diameter & 166 & $\mathrm{~mm}$ \\
Outer diameter & 218 & $\mathrm{~mm}$ \\
Height & 235 & $\mathrm{~mm}$ \\
\hline
\end{tabular}

sidering the mechanical integrity and the operational reliability etc. Although the LTS wire has a better critical current density than the HTS tape, the LTS magnet has the unexpected characteristics such as the training effect. So when the LTS magnet is designed, many other factors must be considered and sometimes the designed results show a difference with the experimental result.

The specifications of a LTS wire for the LTS background magnet with arrangement winding are shown in Table 1. The LTS background magnet is wound with NbTi wire supplied by Oxford Ltd. Fig. 1 shows the arranged LTS background magnet. The LTS background magnet was fabricated using epoxy-impregnated winding. The magnetic field distribution of the fabricated LTS background magnet is shown in Fig. 2. The calculated center magnetic field is $2.17 \mathrm{~T}$ when transport current is $150 \mathrm{~A}$. Fig. 3 shows the magnetic field distribution of the LTS background magnet according to the radial distance and the axial distance from the center.

\subsection{HTS insert coil}

With applying external magnetic field using the LTS background magnet, an electromagnetic field analysis of the HTS insert coil was carried out using MagNet (commercial electromagnetic analysis program), and the magnetic scalar potential method is used to reduce analytical

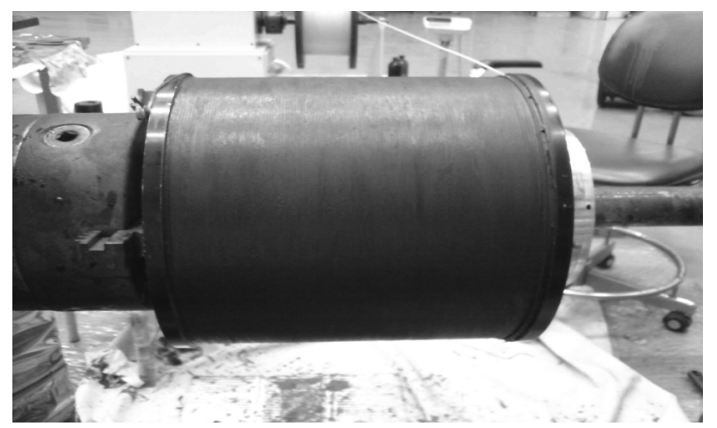

Fig. 1. The arranged LTS background magnet.

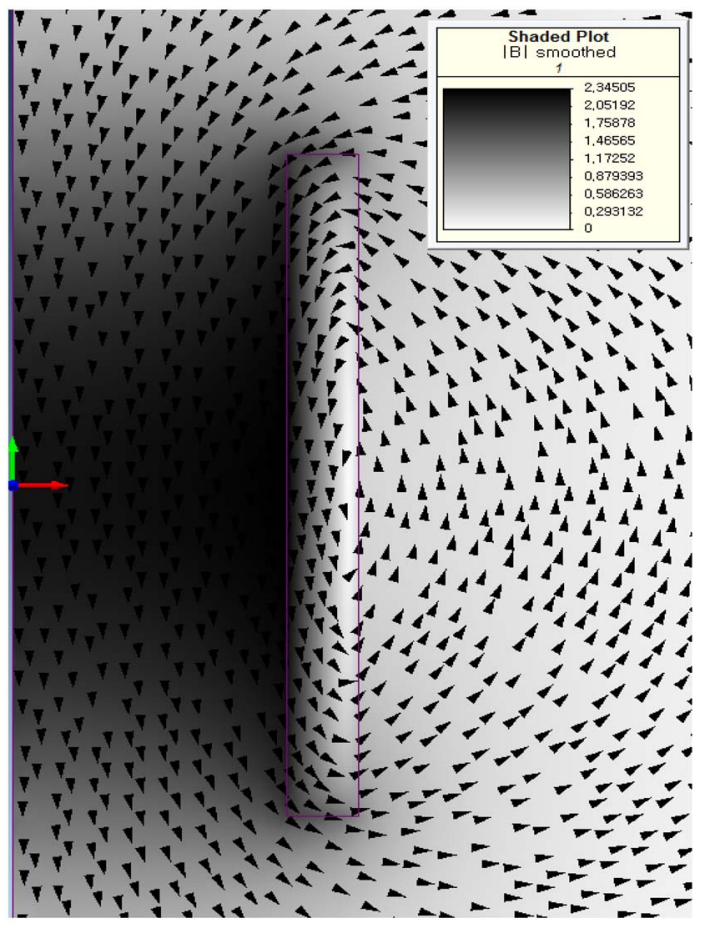

Fig. 2. (Color online) The calculated 2 dimensional magnetic field distribution of the designed LTS background magnet when transport current is $150 \mathrm{~A}$.

time and memory capacity. The 2-dimensional numerical analysis was carried out in order to analyze an electromagnetic field. The 2-dimensional numerical analysis can be classified into two ways, the Cartesian (XY) model and the axis-symmetric (RZ) model. A Cartesian model represents a cross-section of a device that extends infinitely in the line direction and an axis-symmetric model represents a cross-section of a device that is revolved by $360^{\circ}$ around the axis of symmetry (the z-axis). In this paper, the analysis model of the HTS insert coil is set by the RZ model.

The HTS insert coil consists of several pancakes windings wound with YBCO CC tape supplied by Sunam Ltd. The gap between double pancake coils (DPC) is $2 \mathrm{~mm}$. The electromagnetic field analysis was carried out in variation of the number of DPC. The critical current of HTS tapes strongly depends on the perpendicular magnetic field components. Fig. 4 shows the dependence of critical current of the superconducting tape on the perpendicular magnetic field. Because the HTS tape of enough piece length cannot be produced, the HTS insert coil in this paper was fabricated with the HTS tape of three reels. The critical currents of the HTS tape show from 125 A to $140 \mathrm{~A}$ at $77 \mathrm{~K}$ and self-field.

The analysis results as increasing the number of DPC were shown in Table 2. The winding length and the 


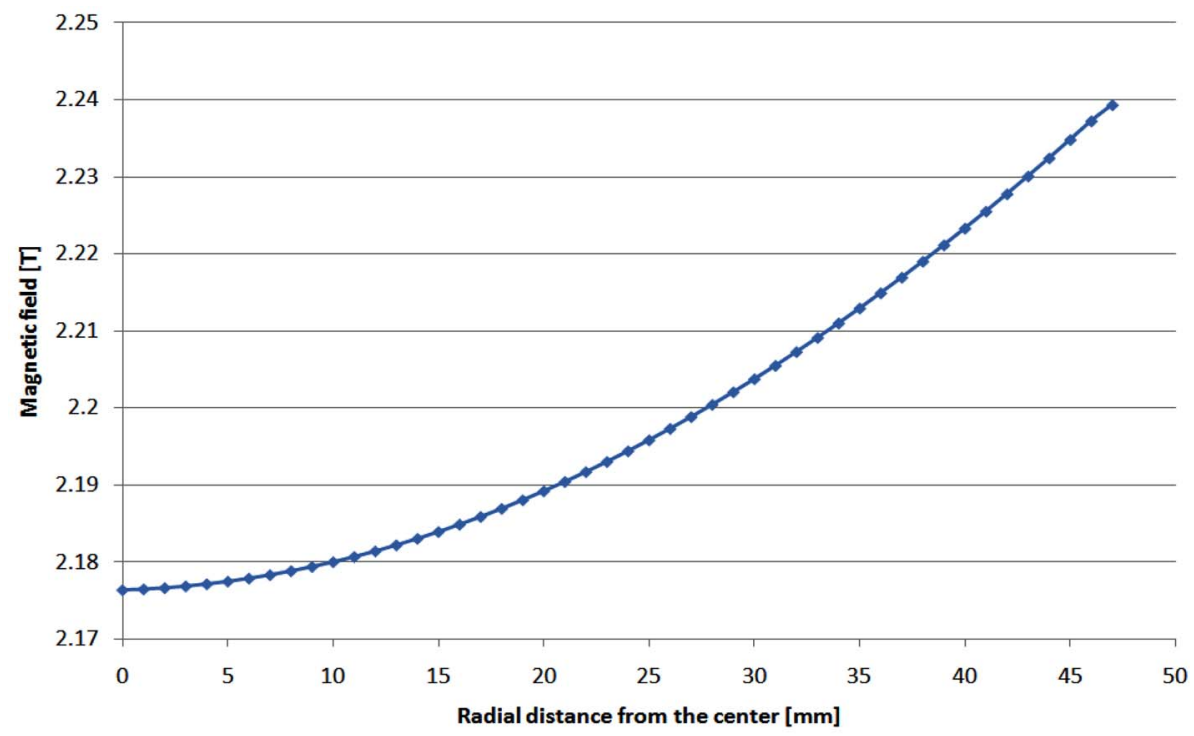

(a)

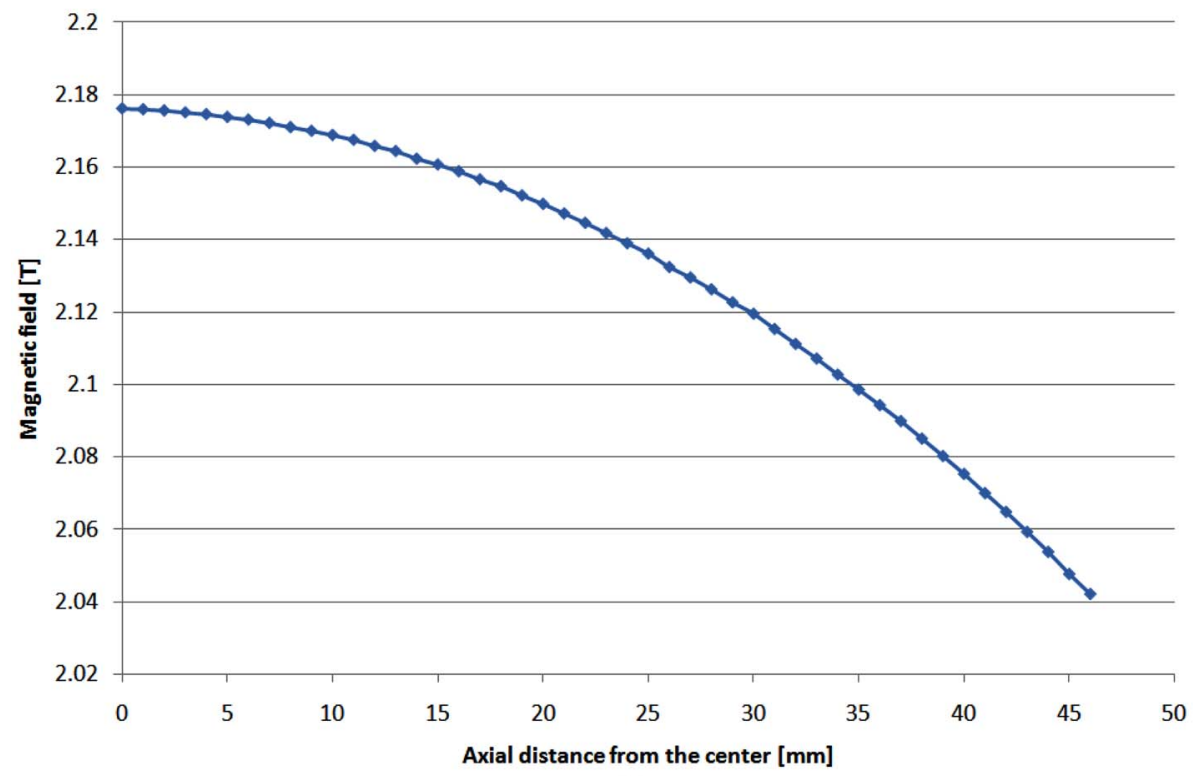

(b)

Fig. 3. (Color online) The magnetic field distribution of the LTS background magnet when transport current is $150 \mathrm{~A}$. (a) according to the radial distance from the center, (b) according to the axial distance from the center.

operating current are fixed at $300 \mathrm{~m}$ and 200 A respectively. As the number of DPC is increased, the field homogeneity gets better, but the center field is decreased. We fabricated the LTS background magnet with $235 \mathrm{~mm}$ height. Because the height of the LTS background magnet is related with the perpendicular magnetic field of the HTS insert coil, the case that the number of DPC is 16 is chosen for HTS insert coil. The HTS insert coil was impregnated with epoxy resin in order to prevent the movement of winding during energizing the magnet.

\section{Preliminary Experiment and Joint}

The HTS insert coil consists of 16 double pancake coils. Before joining between double pancake coils, the critical current of each double pancake coil was measured. Fig. 5 shows the critical current of each double pancake coils. Fig. 5 shows that the critical current of DPC \#1 and DPC \#5 was higher than the others. Because the HTS insert coil is stacking structure, the double pancake coils at the bottom and the top is more suffered than the 


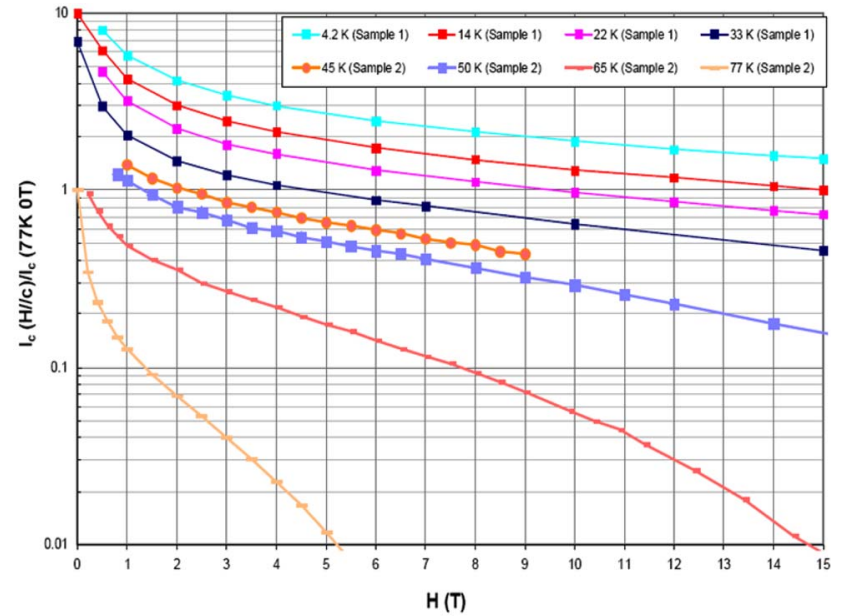

Fig. 4. (Color online) The dependence of critical current of the superconducting tape on the perpendicular magnetic field.

other double pancake coils because of the perpendicular magnetic field. So the DPC \#1 and DPC \#5 are located at the bottom and the top respectively, and the weak DPC \#9 is located at the middle.

The joining technology for the HTS insert coil is considered indispensible to magnet construction because it consists of several stacked pancake coils. Coils are connected each other with low resistance using the lab joining method. The lap joint in this paper was carried out by using a splicing machine. The splicing machine is divided into two parts according to functions. One is a temperature controller. This controller can deal with heating capacity interplaying with thermocouples and a temperature sensor by a PID feedback circuit [6]. The other is heating plate. This consists of a supporter to give heat to the plate and a cover.

\section{Experiment and Result}

The hybrid (HTS/LTS) magnet was tested at liquid helium. The LTS background magnet has with $1.2 \mathrm{~km}$ winding length, $152 \mathrm{~mm}$ inner bore and $260 \mathrm{~mm}$ outer diameter, and $235 \mathrm{~mm}$ height. The HTS insert coil was located inside the LTS background magnet. The HTS

Table 2. The analysis results as increasing the number of DPC when the operating current is $200 \mathrm{~A}$.

\begin{tabular}{|c|c|c|c|c|c|}
\hline \multirow{2}{*}{ Specification } & \multicolumn{5}{|c|}{ The Number of DPC } \\
\hline & 10 & 12 & 14 & 16 & 18 \\
\hline Inductance & 0.0552 & 0.0502 & 0.043 & 0.038 & 0.034 \\
\hline Height (mm) & 108 & 130 & 152 & 174 & 196 \\
\hline Maximum magnetic field & 2.23 & 1.98 & 1.74 & 1.55 & 1.4 \\
\hline Maximum perpendicular field & 1.67 & 1.50 & 1.34 & 1.22 & 1.12 \\
\hline Center field & 1.96 & 1.78 & 1.58 & 1.42 & 1.28 \\
\hline Turns (SPC) & 55 & 47 & 40 & 35 & 31 \\
\hline Homogeneity (\%) & 4.81 & 2.92 & 1.83 & 1.19 & 0.8 \\
\hline
\end{tabular}

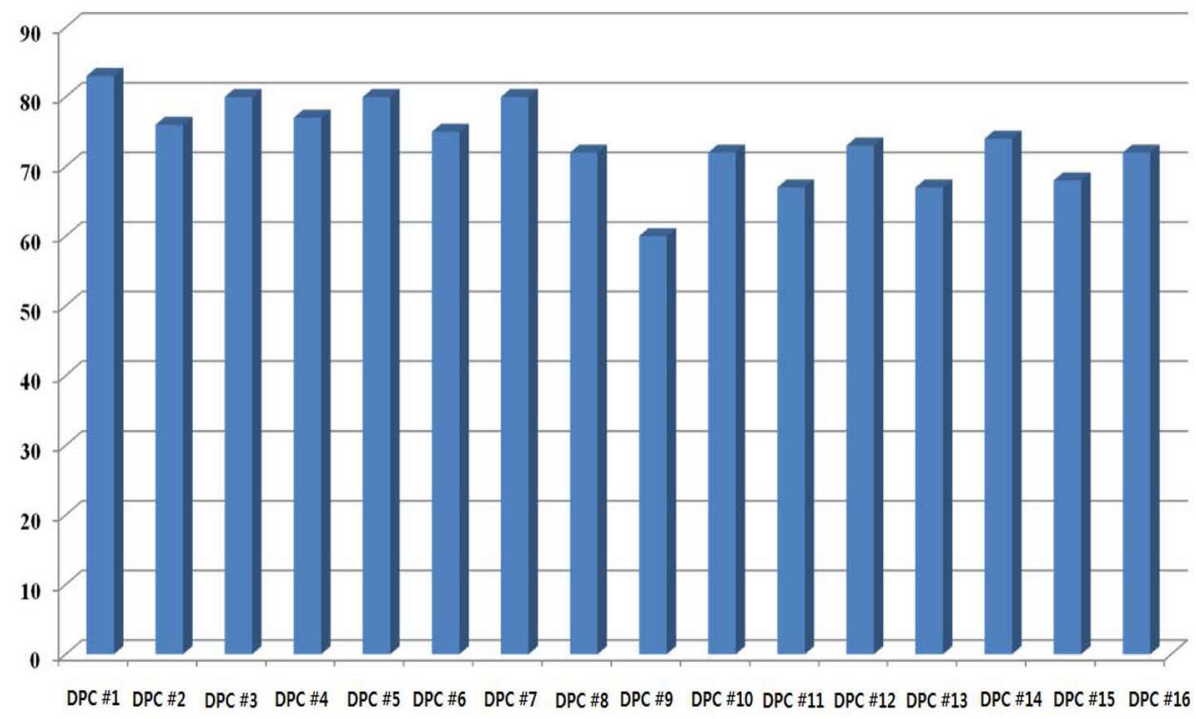

Fig. 5. (Color online) The critical current of each double pancake coils. 


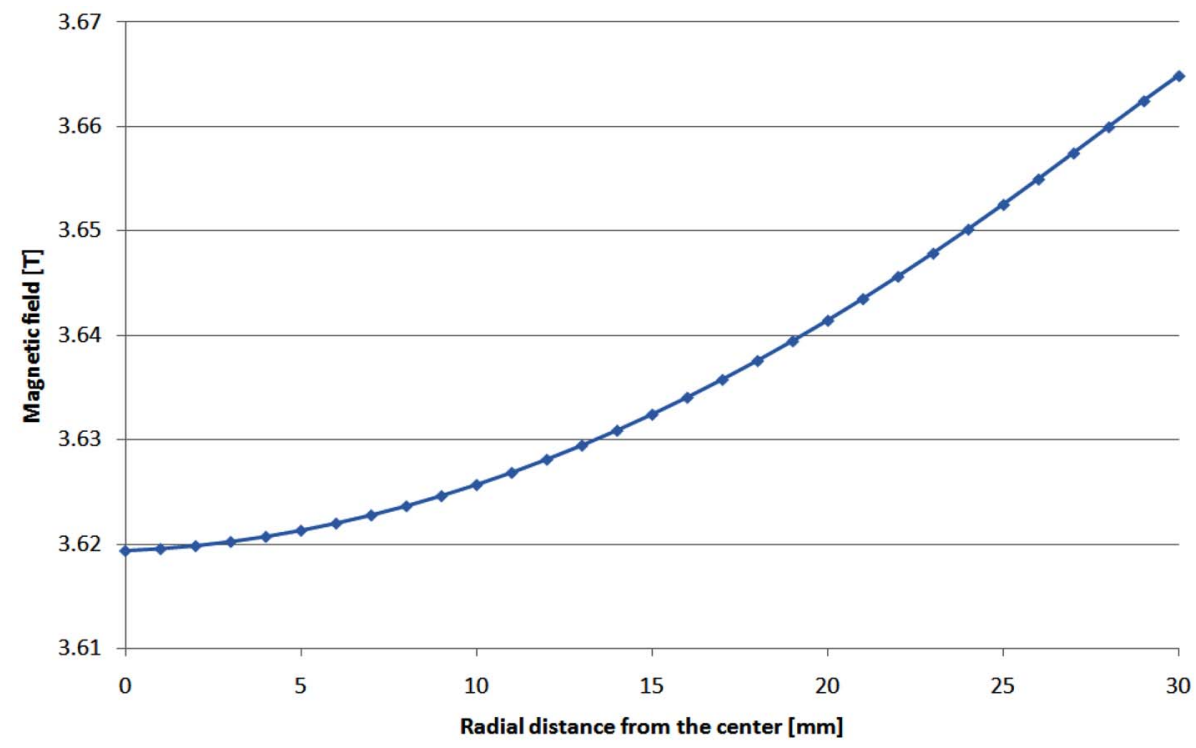

(a)

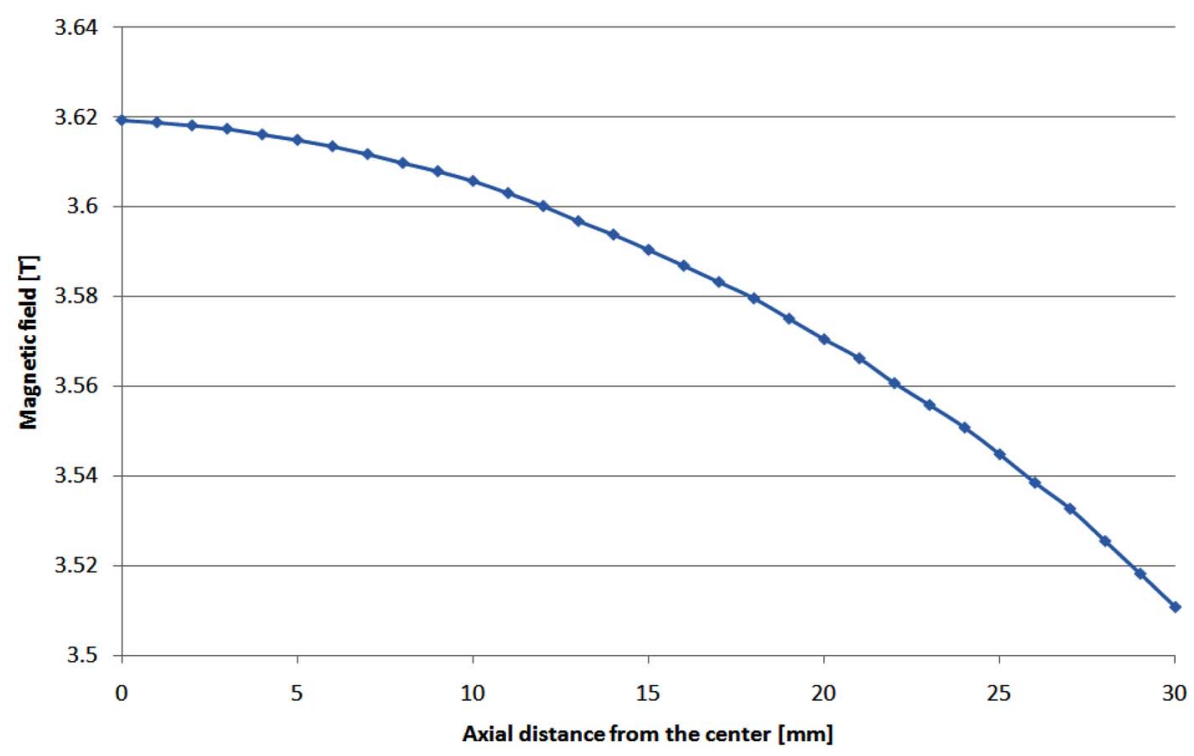

(b)

Fig. 6. (Color online) The calculated magnetic field distribution of the hybrid (LTS/HTS) magnet when operating currents of the LTS background magnet and the HTS insert coil are $150 \mathrm{~A}$ and $200 \mathrm{~A}$ respectively. (a) according to the radial distance from the center, (b) according to the axial distance from the center.

insert coil has with $300 \mathrm{~m}$ winding length, $80 \mathrm{~mm}$ inner diameter and $150 \mathrm{~mm}$ outside diameter. Fig. 6 shows the calculated magnetic field distribution of the hybrid (LTS/ HTS) magnet when operating currents of the LTS background magnet and the HTS insert coil are $150 \mathrm{~A}$ and 200 A respectively. Two power supplies are used for applying a current to LTS background magnet and HTS insert coil. A step-hold method is used for the current ramp for the hybrid magnet test. For the step-hold method, the current values for each step are first determined from 0 to the value above the estimated critical current with a proper interval (step), and the currents held at each step in the stable condition (hold). The magnetic field of the center is measured at each current step. When one power supply is ramping-up state to the some value (step), the other power supply is held at some step (hold). This process can be detected the quench quickly and prevent the damage of the magnet. Fig. 7 shows the current traces of hybrid magnet and the magnetic field of the center.

The expected critical current of the LTS background 


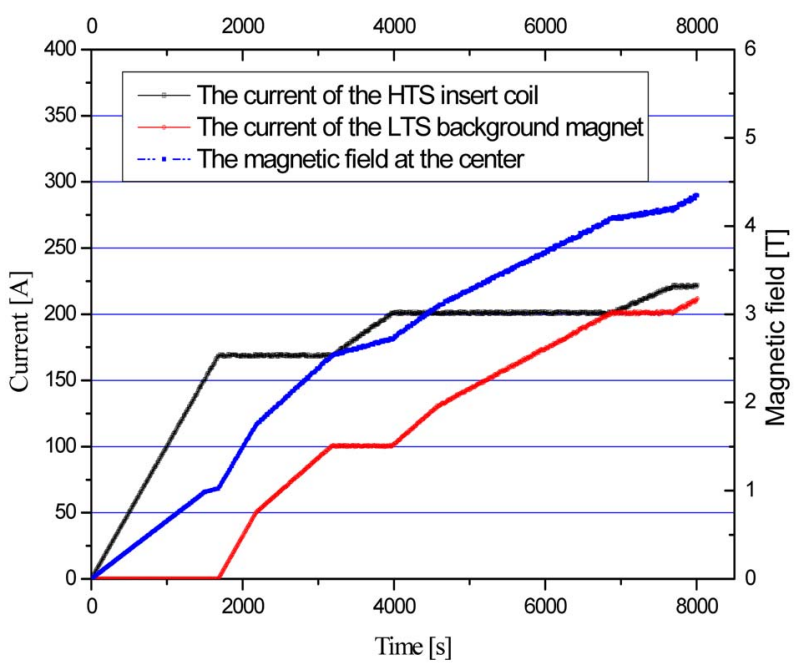

Fig. 7. (Color online) The current traces of hybrid magnet and the magnetic field of the center.

magnet is $150 \mathrm{~A}$. This value was acquired when only the LTS background magnet without the HTS insert coil was operated. However, the experimental result shows that the LTS background magnet can be operated stable beyond 210 A. And the HTS insert coil can be operated stable beyond $220 \mathrm{~A}$. The operating current cannot ramp up any more because of power supply limitation and the hybrid magnet does not experience the quenching state. The final value at the center was $4.32 \mathrm{~T}$. On the LTS background magnet, an incident of premature quench is induced by a mechanical event, primarily either conductor motion or epoxy fracture. A magnet suffering premature quenches generally "training effect" and improves its performance progressively to the point where it finally reaches its intended operating current. The LTS background magnet in this paper experiences the quench at $150 \mathrm{~A}$ at first. But it has not been experience the quench state sufficiently to the final point. On the HTS insert coil, many HTS magnets are designed to have a design margin which is the decision of the operating current below the critical current. A design margin is decided considering the magnet size and the epoxy-resin impregnation and the conductor property etc. The calculated critical current of the HTS insert coil at liquid helium is $470 \mathrm{~A}$, and the operating current $220 \mathrm{~A}$ is a margin of 54 percent. The perpendicular magnetic field in the magnet was analyzed using the electromagnetic analysis, and the critical current of the coil was calculated by using the electromagnetic analysis results and Fig. 4 [7]. Because the superconducting magnet is ordinary designed a margin of 30 percent, the margin of 54 percent seems to be big. As earlier mentioned, the HTS magnet can be easy damaged if quench occurs. Besides, a high operating current at liquid helium temperature can more damage the HTS magnet. Further investigation will be carried out that hybrid magnet does experience the quenching state.

\section{Conclusion}

In this paper, we have made and tested the hybrid (LTS/ HTS) magnet at liquid helium. Considering the field homogeneity, the case that the number of DPC is 16 is suitable for HTS insert coil. The experimental result shows that the LTS background magnet and the HTS insert coil can be operated stable beyond $220 \mathrm{~A}$ and $210 \mathrm{~A}$. The final value $4.32 \mathrm{~T}$ at the center was acquired.

\section{Acknowledgements}

This work was supported by the Korea Science and Engineering Foundation (KOSEF) through the National Research Lab. Program funded by the Ministry of Science and Technology (No. R0A-2009-000-20063-0).

\section{References}

[1] A. Zhukovsky, Y. Iwasa, E. S. Bovrov, J. Ludlam, J. E. C Williams, R. Hirose, and Z. Ping Zhao, IEEE Trans. Magn. 28, 644 (1992).

[2] D. W. Hazelton, Y. Xing, H. W. Weijers, and S. W. Van Sciver, IEEE Trans. Appl. Supercond. 9, 956 (1999).

[3] M. Bechenback, F. Hornung, M. Klaser, P. Leys, B. Lott, and Th. Schneider, IEEE Trans. Appl. Supercond. 15, 1484 (2005).

[4] K. Tasaki, M. Ono, T. Kuriyama, M. Kyoto, S. Hanai et al., IEEE Trans. Appl. Supercond. 15, 1512 (2005).

[5] T. Takeuchi, H. Kitaguchi, N. Banno, Y. Iijima, A. Kikuchi, K. Tagawa, Y. Suzuki, M. Yoshikawa, and S. Hayashi, IEEE Trans. Appl. Supercond. 17, 2684 (2007).

[6] K. S. Chang, D. K. Park, S. E. Yang, H. C. Jo, H. J. Kim, Y. S. Yoon, H. S. Kim, and T. K. Ko, IEEE Trans. Appl. Supercond. 20, 1577 (2010).

[7] S. Choi, J.-H. Bae, M.-H. Sohn, W.-S. Kim, C. Park, J.-K. Lee, S. Lee, and K. Choi, IEEE Trans. Appl. Supercond. 19, 1249 (2009). 